\title{
NOTA
}

\section{AVALIAÇÃO DA DISPONIBILIDADE DE MANGANÊS E FERRO EM SOLOS PELO USO DO MÉTODO MODIFICADO DA RESINA DE TROCA IÔNICA(1)}

\author{
C. A. ABREU (2), B. VAN RAIJ (3), M. F. ABRE U(2) \& A. PAZ GONZALEZ ${ }^{(4)}$
}

\begin{abstract}
RESUMO
A avaliação do método modificado da resina de troca de íons para extração de Mn e Fe dos solos foi o objetivo desta pesquisa. Foram utilizadas 44 amostras de solo, cujos teores de Mn variaram de baixos a altos e de Fe de médios a altos. Como plantas-teste, utilizaram-se o milho e a soja, cultivadas em casa de vegetação. O Fe e o Mn do solo foram determi nados, usando o método modi ficado da resina de troca de íons, DTPA, AB-DTPA, Mehlich-1 e Mehlich-3. Os coeficientes de correlação entre Mn no solo e Mn acumulado na parte aérea da soja foram: resina $\left(0,62^{*}\right)$, DTPA $\left(0,58^{*}\right)$, Mehlich-3 $\left(0,54^{*}\right)$, Mehlich-1 $\left(0,51^{*}\right)$ e ABDTPA (0,26NS). Para o milho, houve correlação entre Mn-solo e Mn-planta somente nas amostras de solo com baixos teores desse elemento, para todos os extratores, exceto para AB-DTPA. Concluiu-se que a resina foi tão eficiente quanto os extratores DTPA, M-1 e M-3 em avaliar a disponibilidade de Mn para a soja, e que nenhum extrator foi eficiente em avaliar a disponibilidade de $\mathrm{Fe}$ para as plantas de milho e soja, com $\mathbf{5 3}$ dias de idade, cultivadas em casa de vegetação.
\end{abstract}

Termos de indexaçao: análise de solo, micronutriente, DTPA, AB-DTPA, Mehlich-1, Mehlich-3.

\section{SUMMARY: EVALUATION OF MANGANESE AND IRON AVAILABILITY IN SOILS BY A MODIFIED ION EXCHANGE RESIN METHOD}

The objective of this research was to evaluatea modified ion exchangeresin method for $\mathrm{Mn}$ and Feextraction from soils. Forty-four soil samples presenting low, medium, and high $\mathrm{Mn}$ and medium to high Fe concentrations were used. Maize and soybeans plants were

(1) Recebido para publicação em junho de 2003 e aprovado em março de 2004

(2) Pesquisadora Científica, Centro de Solos e Recursos Agroambientais, Instituto Agronômico - IAC. Caixa Postal 09, CEP 13001970 Campinas ( SP). Bolsista do CNPq. E-mail: cleide@iac.sp.gov.br

(3) Pesquisador Científico, IAC. E-mail: bvanraij@iac.sp.gov.br

(4) Professor do Departamento de Edafologia, Universidade A Coruña, A Zapateira, s/n. CEP 15071 A Coruña (Espanha). E-mail: tucho@udc.es 
grown as test plants in a pot experiment in a greenhouse. Soil samples were analyzed for $\mathrm{Mn}$ and Feusing the modified ion exchange resin, DTPA, AB-DTPA, Mehlich-1, and Mehlich-3 methods. The correlation coefficients between soil $\mathrm{Mn}$ and $\mathrm{Mn}$ accumulated in the soybean shoot were: ion exchangeresin $\left(0.62^{*}\right)$, DTPA $\left(0.57^{*}\right)$, Mehlich-3 $\left(0.54^{*}\right)$, Mehlich-1 $\left(0.51^{*}\right)$, and AB-DTPA (0.26). For maize, significant corredation coefficients between soil $\mathrm{Mn}$ and plant-Mn were obtained only when low-initial $\mathrm{Mn}$ soil samples were considered for all extractants, except for AB-DTPA. For soybeans, the modified ion exchange resin method was as efficient as DTPA, Mehlich- 1 and Mehlich-3 for theevaluation of soil Mn availability. None of the methods was efficient for eval uating the soil Feavailability for the 53 day-old maizeand soybean plants grown in greenhouse.

Index terms: soil test, extractants, DTPA, AB-DTPA, Mehlich-1, Mehlich-3.

\section{INTRODUÇAO}

Com a evolução e o crescimento da agricultura brasileira, os micronutrientes, principalmente o Fe e o $\mathrm{Mn}$, tornam-se cada vez mais importantes e merecedores de atenção da pesquisa, tanto em casos de aparecimento de deficiências como na ocorrência de toxidez.

A análise desoloé complexa para Mn eFeporque a concentração desses el ementos é muito influenciada pelas reações de oxirredução. Os coeficientes de correlação entre os teores de $\mathrm{Mn}$ no solo e na planta, obtidos com extratores ácidos ou quelantes, são, em sua maioria, baixos. As soluções salinas, tamponadas ou não, que determinam preferencialmentea forma trocável de $M n$, têm sido mais eficientes em avaliar a disponibilidade desse elemento para as plantas (Muraoka et al., 1983; Abreu et al., 1994b). Muraoka et al. (1983) encontraram valores de correlação entre o Mn extraído do solo e as quantidades de $\mathrm{Mn}$ na planta de 0,93** ( $\mathrm{NH}_{4} \mathrm{OAC}$ ), 0,46* (DTPA) e 0,21NS (EDTA). Abreu et al. (1994b) constataram que, dentre as soluções testadas (Mehlich-1, $\mathrm{HCl}, \mathrm{H}_{3} \mathrm{PO}_{4}$, DTPA, resina, $\mathrm{NH}_{4} \mathrm{OAc}, \mathrm{NH}_{4} \mathrm{OAc}$ + hidroquinona e $\mathrm{CaCl}_{2}$ ), a de cloreto de cálcio estava entre os métodos que melhor estimaram a disponibilidade de $\mathrm{Mn}$ frente às mudanças de $\mathrm{pH}$.

Pesquisas realizadas no Brasil relacionadas com a sel eção de extratores para avaliar a disponi bilidade de Fe são escassas. A maior parte dos trabalhos não leva em consideração a absorção de Fe pelas plantas. Dentre os poucos trabal hos que usaram a planta como indicadora da disponibilidade de Fe, citam-se os de Defelipo et al. (1991) e Amaral Sobrinho et al. (1993). Esses últimos autores obtiveram um coeficiente de correlação entre Fe no solo e Fe no sorgo de 0,92* (Mehlich-1) e de 0,65* (DTPA).

A proposta do uso de resinas de troca iônica em análise de solo é bastante antiga, principalmente para $\mathrm{P}$. Além de $\mathrm{P}, \mathrm{K}, \mathrm{Ca}, \mathrm{Mg}$ e Na, a resina de troca deíons poderá ser utilizada para aval iação de $\mathrm{S}, \mathrm{Cu}$, Mo, Cd, Pb e Mn. Usando como planta-teste a soja, Abreu et al . (1994a) encontraram valores de coeficientes de correlação entre os teores de $M n$ no solo e na parte aérea das plantas de 0,65** (Mehlich-1), 0,64** (resina) e 0,51** (DTPA). Em trabalho subseqüente com soja, esses coeficientes de correlação foram de: 0,79* (resina), 0,45NS (Mehlich-1) e 0,40NS (DTPA) (Abreu et al., 1994b). Contudo, quando os teores dos micronutrientes são muito baixos, torna-sedifícil a quantificação pela resina por causa da larga relação solo:solução extratora $\left(2,5 \mathrm{~cm}^{3}: 50 \mathrm{~mL}\right)$. Tentando solucionar esse problema, Almei da (1999) propôs o uso de uma relação solo : solução extratora de 2,5 cm $\mathrm{cm}^{3}: 25 \mathrm{~mL}$ ea adição de citrato de amônio na solução extratora da resina (ácido clorídrico mais cloreto de amônio). O autor verificou que a união dos dois processos de extração, um de troca iônica e outro de complexação, permitiu uma redução no volume de sol ução extratora e a possibilidade de se estender o método para a determinação de Mn, Fee $\mathrm{S}$.

Com base nessas informações, foi realizada esta pesquisa, queteve o objetivo decomparar a eficiência do método modificado da resina de troca de íons (adição do citrato de amônio à solução extratora ácido clorídrico + cloreto de amônio) para extrair o $\mathrm{Mn}$ e o $\mathrm{Fe}$ disponíveis do solo com os métodos tradicionalmente usados (DTPA, AB-DTPA, Mehlich-1 e Mehlich-3).

\section{MATERIAL E MÉTODOS}

Dois experimentos foram realizados em casa de vegetação, utilizando amostras retiradas na profundidade de 0-20 cm de quarenta equatro solos do estado de São Paulo representativos das classes Oxisol, Ultisol, Alfisol, I nceptsol e Entisol.

As amostras foram secas ao ar e passadas em peneira de malha com abertura de $3 \mathrm{~mm}$. Quando necessário, fez-se a calagem, usando $\mathrm{Ca}(\mathrm{OH})_{2}$ e $\mathrm{MgCO}_{3}$, na proporção de 4 moles de Ca para 1 de Mg, para el evar a saturação por bases para $70 \%$. Todas as amostras permaneceram incubadas por um período de 16 dias, mantendo-se a umidade a $60 \%$ da capacidade de campo, por meio de pesagens diárias dos vasos. 
Após o período deincubação, fez-se uma adubação da seguinte forma: (a) o $P$ foi misturado ao solo na forma de superfosfato triplo, nas doses de 358, $179 \mathrm{e}$ $89,5 \mathrm{mg} \mathrm{dm}^{-3}$ de $\mathrm{P}$, respectivamente, para sol os com teores baixos, médios e altos de $\mathrm{P}$ extraídos pela resina (Raij et al., 1997); (b) K, N e S foram adicionados ao solo nas formas de nitrato de $K$, sulfato de $K$ e sulfato de amônio, para fornecer $100 \mathrm{mg} \mathrm{dm}^{-3}$ de K, $46 \mathrm{mg} \mathrm{dm}^{-3}$ de N e $20 \mathrm{mg} \mathrm{dm}^{-3}$ de $\mathrm{S}$, e (c) B, Cu eZn foram aplicados no solo, na forma de solução, nas amostras que apresentavam teores baixos desses el ementos, extraídos com a solução de DTPA pH 7,3. As doses, em $\mathrm{mg} \mathrm{dm}^{-3}$, foram de 0,5 de $B, 1,0$ de $C u$ e 2,0 de $Z n$, nas formas de ácido bórico, sulfato de $\mathrm{Cu}$ e sulfato de $\mathrm{Zn}$, respectivamente.

Dentre as 44 amostras de solo col etadas, não se adicionou $\mathrm{Mn}$ em 37 com teores médios a altos de $M n\left(=1,3 \mathrm{mg} \mathrm{dm}^{-3}\right.$ pelo DTPA). As outras sete amostras, cujos teores de $\mathrm{Mn}$ eram baixos $\left(<1,3 \mathrm{mg} \mathrm{dm}^{-3}\right)$, foram divididas em dois grupos: um sem adição de $\mathrm{Mn}$ e outro que recebeu $15 \mathrm{mg} \mathrm{dm}^{-3}$ de $\mathrm{Mn}$ na forma de cl oreto de manganês. Para oFe, não foi feita a adição, pois todas as 44 amostras apresentaram teores médios a altos $\left(=5 \mathrm{mg} \mathrm{dm}^{-3}\right)$. Todas as unidades experimentais permaneceram incubadas por um período de 15 dias, mantendo-se a umidade a $60 \%$ da capaci dade de campo, por meio da pesagem diárias dos vasos.

Procedeu-se à semeadura do milho, utilizando 10 sementes por vaso com capacidade de $2 \mathrm{dm}^{-3} \mathrm{e}$ deixando-se, após o desbaste, três plantas por vaso. A adubação de cobertura foi parcelada em três vezes, usando o nitrato de amônio na primeira e o nitrato de Ca nas demais. Foi utilizada a dose de $25 \mathrm{mg} \mathrm{dm}^{-3}$ de $\mathrm{N}$ em cada aplicação. Durantetodo o experimento, procurou-se manter a umidade do sol o a $70 \%$ da capacidade de campo, por adições diárias de água destilada, controladas por meio da pesagem dos vasos.

Aos 53 dias da emergência, realizou-seo corteda parte aérea das plantas de milho, e as raízes foram separadas do solo e eliminadas. O material vegetal foi lavado, seco em estufa com circulaçao forçada de ar a $70^{\circ} \mathrm{C}$, até peso constante, para determinação da matéria seca, e após, moído e digerido por via seca (Bataglia et al., 1983). Determinaram-se os teores de $\mathrm{Mn}$ e $\mathrm{Fe}$ nos extratos, utilizando um espectrômetro de emissão por plasma (ICP-AES).

Em seguida, fez-se um segundo cultivo nos mesmos vasos usados anteriormente com milho, utilizando-se quatro plantas de soja por vaso. Esse ensai o foi semel hante ao adotado para o milho.

Antes de cada cultivo, retirou-se uma amostra de solo de cada unidade experimental, que foi seca ao ar e passada em peneira de malha de $2 \mathrm{~mm}$, para análise de $\mathrm{Mn}$ e Fe, utilizando cinco extratores, descritos a seguir:
Extração com resina de troca iônica, método modificado por Almeida (1999): as etapas de preparação da resina trocadora de íons (pré-condicionamento, tratamento para uso e recuperação) foram as mesmas recomendadas por Raij et al. (1987). As modificações no procedimento de extração estão descritas a seguir: $2,5 \mathrm{~cm}^{3}$ de solo +25 mL deágua +uma bola de vidro foram agitados por 30 min a 220 rpm. Logo após, retirou-se a bola de vidro e adicionaram-se $2,5 \mathrm{~cm}^{3}$ de resina trocadora (Amberlite IRA-400 e Dowex 50-W, na proporção de 1:1 v/v) mantendo-se em agitação por $16 \mathrm{~h}$ a $220 \mathrm{rpm}$. Após a separação da resina, foram adicionados $25 \mathrm{~mL}$ da solução extratora $(\mathrm{HCl}$ $0,25 \mathrm{~mol} \mathrm{~L}^{-1}+\mathrm{NH}_{4} \mathrm{Cl} 0,75 \mathrm{~mol} \mathrm{~L}^{-1}+\left(\mathrm{NH}_{4}\right)_{2} \mathrm{HC}_{6} \mathrm{H}_{5} \mathrm{O}_{7}$ 0,30 $\mathrm{mol} \mathrm{L}^{-1}$ ) e agitados por uma hora. O extrato foi utilizado para as determinações de Fee $\mathrm{Mn}$.

Extração com DTPA: dietilenotriaminopentaacético + trietanolamina na presença de cl oreto deCa (DTPA 0,005 mol L-1 +TEA 0,1 mol L-1 $+\mathrm{CaCl}_{2}$ $0,01 \mathrm{~mol} \mathrm{~L}^{-1}$ a pH 7,3), segundo Lindsay \& Norvell (1978) - $10 \mathrm{~cm}^{3}$ de solo $+20 \mathrm{~mL}$ de solução extratora, agitação por $2 \mathrm{~h}$.

Extração com AB-DTPA: dietilenotriaminopentaacético + bicarbonato de amônio $\left(\mathrm{NH}_{4} \mathrm{HCO}_{3}\right.$ $1 \mathrm{~mol} \mathrm{~L}^{-1}+$ DTPA 0,005 mol L-1 a pH 7,6), de acordo com Soltanpour et al . (1977) - $10 \mathrm{~cm}^{3}$ de sol o $+20 \mathrm{~mL}$ da solução extratora, agitação por $15 \mathrm{~min}$.

Extração com Mehlich-1: $\mathrm{HCl} 0,05 \mathrm{~mol} \mathrm{~L}^{-1}+$ $\mathrm{H}_{2} \mathrm{SO}_{4} 0,0125 \mathrm{~mol} \mathrm{~L}^{-1}$, conforme Mehlich (1953) $5 \mathrm{~cm}^{3}$ de solo $+25 \mathrm{~mL}$ de solução extratora, agitação por 30 min.

Extração com Mehlich-3: $\left(\mathrm{CH}_{3} \mathrm{COOH} 0,2 \mathrm{~mol} \mathrm{~L}-1\right.$ $+\mathrm{NH}_{4} \mathrm{NO}_{3} 0,25 \mathrm{~mol} \mathrm{~L}^{-1}+\mathrm{NH}_{4} \mathrm{~F}^{2} 0,015 \mathrm{~mol} \mathrm{~L}^{-1}+$

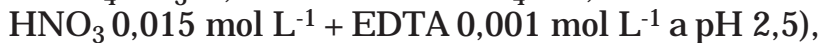
descrita por Mehlich (1984) $-5 \mathrm{~cm}^{3}$ de solo $+20 \mathrm{~mL}$ da solução extratora, agitação por 5 min.

O cál culo de correlação simples foi feito entre os teores de $\mathrm{Mn}$ ou $\mathrm{Fe}$ no solo ( $\mathrm{Mn}$-solo e Fe-solo) extraídos pelas diferentes soluções e os teores ou conteúdos (quantidade acumulada) de $\mathrm{Mn}$ ou Fe no tecido vegetal ( $\mathrm{Mn}$-planta ou Fe-planta) para o milho e para a soja. Calcularam-setambém as correlações entre os teores no solo obtidos para os diferentes métodos quími cos utilizados para análise de Mn eFe.

\section{RESULTADOS E DISCUSSÃO}

\section{Manganês}

Durante os ensaios, não foram observados sintomas visuais de deficiência de $M n$, embora al gumas amostras de sol os apresentassem teores de Mn baixos, de 0,9 a 1,2 $\mathrm{mg} \mathrm{dm}^{-3}$, extraídos pelo DTPA, conforme interpretação de Raij et al. (1997). Nessas amostras de solos, as concentrações de $\mathrm{Mn}$ na parte aérea variaram de 29,2 a 104,3 mg kg-1, 
para a soja, e de 66,5 a $82,5 \mathrm{mg} \mathrm{kg}^{-1}$, para o milho, as quais são consideradas adequadas para o desenvolvimento dessas culturas (Raij et al., 1997). Nas demais amostras, a amplitude de variação foi de 16,6 a $180 \mathrm{mg} \mathrm{kg}^{-1}$, para a soja, e de 19 a $365 \mathrm{mg} \mathrm{kg}^{-1}$, para o milho. As plantas de soja que apresentaramteores deMn de16,6a 16,8 $\mathrm{mg} \mathrm{kg}^{1}$, abaixo dos valores críticos para folhas, foram as dos tratamentos em solos cujos teores de $\mathrm{Mn}$ eram de 1,6 e 1,9 $\mathrm{mg} \mathrm{dm}^{-3}$, extraídos pelo DTPA.

As soluções ácida (Mehlich-1) e mista (ácida/ quelante - Mehlich-3), extraíram mais $\mathrm{Mn}$ que as quelantes (AB-DTPA eDTPA) equea resina de troca iônica, tanto no solo cultivado com soja como naquele com milho (Quadro 1). A maior extração pelas sol uções, ácida e mista, pode ser devida à sua maior capacidade em sol ubilizar o M n ligado aos óxidos de Fe. No cultivo da soja, as mel hores correl ações entre o Mn extraído do solo pel os di ferentes métodos foram obtidas entre Mn-Mehlich-1 e Mn-Mehlich-3 ( $r=$ $0,98^{* *}$ ) e entre Mn-DTPA com Mn-Mehlich-1 ( $r=$ $0,96 * *$ ) ou resina ( $r=0,94 *$ ) (Quadro 2 ). As piores correlações foram observadas entre $\mathrm{Mn}$-Mehlich-1 e Mn-AB-DTPA ( $r=0,29 \mathrm{NS})$ e entre Mn-AB-DTPA eMn-DTPA ( $r=0,27 \mathrm{NS})$.

Quadro 1. Amplitude de variação dos teores de ferro e manganês extraídos por diferentes métodos em amostras de solo retiradas antes do cultivo da soja e do milho

\begin{tabular}{|c|c|c|c|c|c|c|c|c|c|c|}
\hline \multirow{2}{*}{ Amplitude } & \multicolumn{2}{|c|}{ Resina } & \multicolumn{2}{|c|}{ DTPA } & \multicolumn{2}{|c|}{ AB-DTPA } & \multicolumn{2}{|c|}{ Mehlich-1 } & \multicolumn{2}{|c|}{ Mehlich-3 } \\
\hline & $\mathbf{F e}$ & Mn & $\mathbf{F e}$ & Mn & $\mathbf{F e}$ & Mn & $\mathbf{F e}$ & Mn & $\mathbf{F e}$ & Mn \\
\hline & \multicolumn{10}{|c|}{$-m g d m^{-3}$} \\
\hline & \multicolumn{10}{|c|}{ Soja } \\
\hline $\begin{array}{l}\text { Mínimo } \\
\text { Máximo }\end{array}$ & $\begin{array}{r}7 \\
33\end{array}$ & $\begin{array}{l}3,3 \\
87\end{array}$ & $\begin{array}{r}5 \\
191\end{array}$ & $\begin{array}{l}1,0 \\
81\end{array}$ & $\begin{array}{r}10 \\
212\end{array}$ & $\begin{array}{c}1,7 \\
56\end{array}$ & $\begin{array}{r}7 \\
127\end{array}$ & $\begin{array}{r}2,8 \\
148\end{array}$ & $\begin{array}{r}12 \\
216\end{array}$ & $\begin{array}{r}3,1 \\
189\end{array}$ \\
\hline \multirow[t]{2}{*}{ Média } & 14 & 18,5 & 33 & 12,5 & 61 & 11,9 & 39 & 30,5 & 89 & 38,2 \\
\hline & \multicolumn{10}{|c|}{ Milho } \\
\hline Mínimo & 10 & 4,3 & 5 & 1,4 & 15 & 2,7 & 8 & 4,1 & 32 & 3,1 \\
\hline Máximo & 43 & 259 & 145 & 165 & 246 & 119 & 246 & 216 & 404 & 189 \\
\hline Média & 18 & 27,2 & 27 & 17,2 & 55 & 24,9 & 55 & 50 & 139 & 38,2 \\
\hline
\end{tabular}

Quadro 2. Coeficientes de correlação entre o manganês extraído do solo por diferentes métodos em 44 amostras de solo retiradas antes do cultivo da soja e do milho com o manganês acumulado na planta ou sua concentração na parte aérea

\begin{tabular}{|c|c|c|c|c|c|c|}
\hline \multirow{2}{*}{ Método } & \multicolumn{2}{|c|}{ Mn-planta } & \multicolumn{4}{|c|}{ Método } \\
\hline & Acumulado & Concentrado & Resina & DTPA & AB-DTPA & Mehlich-1 \\
\hline & mg vaso-1 & $\mathrm{mg} \mathrm{kg}^{-1}$ & 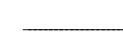 & 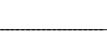 & $n^{-3}$ & 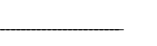 \\
\hline & & & & & & \\
\hline Resina & $0,62 *$ & $0,49 *$ & & & & \\
\hline DTPA & $0,57 *$ & $0,48^{*}$ & $0,94 * *$ & & & \\
\hline AB-DTPA & $0,26 \mathrm{NS}$ & $0,18^{\mathrm{NS}}$ & 0,38 NS & $0,27^{\mathrm{NS}}$ & & \\
\hline Mehlich-1 & $0,51 *$ & $0,42 *$ & $0,89 * *$ & $0,96 * *$ & $0,29^{\text {NS }}$ & \\
\hline \multirow[t]{2}{*}{ Mehlich-3 } & $0,54 *$ & $0,44 *$ & $0,89 * *$ & $0,94 * *$ & $0,30^{\mathrm{NS}}$ & $0,98 * *$ \\
\hline & \multicolumn{6}{|c|}{ Milho } \\
\hline Resina & $0,22^{\mathrm{NS}}$ & $0,17^{\mathrm{NS}}$ & & & & \\
\hline DTPA & $0,20^{\text {NS }}$ & $0,15^{\mathrm{NS}}$ & $0,94^{* *}$ & & & \\
\hline AB-DTPA & $0,14^{\mathrm{NS}}$ & $0,13^{\mathrm{NS}}$ & $0,82 * *$ & $0,86 * *$ & & \\
\hline Mehlich-1 & $0,15^{\mathrm{NS}}$ & $0,14^{\mathrm{NS}}$ & $0,77^{* *}$ & $0,83 * *$ & $0,93 * *$ & \\
\hline Mehlich-3 & $0,20^{\text {NS }}$ & $0,19^{\text {NS }}$ & $0,79 * *$ & $0,85^{* *}$ & $0,89 * *$ & $0,98 * *$ \\
\hline
\end{tabular}

*; ** $\mathrm{e}^{\mathrm{NS}}$ : Significativos a 1 e 5\% e não significativo. 
Para a cultura da soja, responsiva ao $\mathrm{Mn}$, foram obtidas correlações positivas entre $\mathrm{Mn}$ no sol o e $\mathrm{Mn}$ na planta para todos os métodos, com exceção do AB-DTPA (Quadro 2). A resina detroca iônica deve estar quantificando preferencialmente oMn trocável do solo, além de outras formas lábeis, como o $\mathrm{Mn}$ facilmentereduzível. A agitação do solo com a resina durante toda a noite, em frasco fechado, pode ter liberado formas de $\mathrm{Mn}$ facilmente reduzíveis. Conforme Goldberg \& Smith (1984), grande parte do $\mathrm{Mn}$ absorvido pelas plantas encontra-se ligado aos sítios de troca de cátions do solo, por atração el etrostática, em equilíbrio direto e rápido com a solução do solo, podendo ser permutado com outros cátions em quantidades estequiométricas. Por isso, as soluções salinas que quantificam o Mn trocável são eficientes em avaliar a disponibilidade desse el emento no solo, como foi observado para o $\mathrm{NH}_{4} \mathrm{OAC}$ (Muraoka et al., 1983) e $\mathrm{CaCl}_{2}$ (Abreu et al., 1994b).

Para o milho, nenhum método foi eficiente em avaliar a disponibilidade de $\mathrm{Mn}$ quando todas as 44 amostras foram utilizadas no cálculo da correlação entre M n-solo e M n-planta (concentração ou acumulado na parte aérea) (Quadro 2). Os valores de coeficiente de correlação entre $\mathrm{Mn}$-solo e $\mathrm{Mn}$ planta foram muito baixos e não-significativos $(<0,22)$. A inclusão dos teores de matéria orgânica e da granulometria nos cálculos da regressão, ou a separação dos solos de acordo com esses atributos, não melhorou a equação de predição da disponibilidade de $\mathrm{Mn}$ na parte aérea do milho.

Todos os métodos foram eficientes em detectar os teores de $\mathrm{Mn}$ no solo modificados pela aplicação de cloreto de Mn. Calculando a correlação somente para amostras de solo que apresentavam teores baixos de $\mathrm{Mn}$ e que receberam ou não adição desse el emento, observaram-se val ores dos coeficientes de correlação entre $\mathrm{Mn}$ no solo e concentração de $\mathrm{Mn}$ na parte aérea do milho de: $0,80^{*}$ (resina), $0,76^{*}$ (Mehlich-3), 0,73* (Mehlich-1), 0,66* (DTPA) e 0,47NS (AB-DTPA). Rosolem et al. (1992), analisando a relação entre $\mathrm{Mn}$ no solo e a concentração de $\mathrm{Mn}$ na fol ha de soja, observaram que, quando os teores de $\mathrm{Mn}$ no solo foram modificados pela aplicação de sulfato de $M n$, tanto o DTPA $\left(r=0,72^{*}\right)$ como o Mehlich-1 $\left(0,68^{*}\right)$ foram eficientes.

\section{Ferro}

Os métodos químicos foram ineficientes em avaliar a disponibilidade de Fe tanto para a cultura da soja como para a do milho (Quadro 3). Em nenhuma situação, houve correl ação entre o teor de Fe no solo e sua concentração na planta ou o seu acúmulo na parte aérea.

Sintomas de deficiência ou toxidez deFena parte aérea não foram observados em nenhuma cultura. A concentração de Fe variou de 63 a $164 \mathrm{mg} \mathrm{kg}^{-1}$ na parte aérea do milho e de 63 a $112 \mathrm{mg} \mathrm{kg}^{-1}$ na soja, teores considerados adequados (Raij et al., 1997). A ausência de sintomas visuais de deficiência pode ser justificada pelo fato de as amostras de solo apresentarem teores médios a altos de $\mathrm{Fe}$ ( $\geq 5 \mathrm{mg} \mathrm{dm}^{-3}$ DTPA) (Quadro 1).

Os métodos tiveram capacidades extrativas diferentes, tendo o Mehlich-3 apresentado a maior extração de Fe e a resina a menor (Quadro 1). A

Quadro 3. Coeficientes de correlação entre o ferro extraído do solo por diferentes métodos em amostras de solo retiradas antes do cultivo da soja e do milho com o ferro acumulado na planta ou sua concentração na parte aérea

\begin{tabular}{|c|c|c|c|c|c|c|}
\hline \multirow{2}{*}{ Método } & \multicolumn{2}{|c|}{ Fe-planta } & \multicolumn{4}{|c|}{ Método } \\
\hline & Acumulado & Concentrado & Resina & DTPA & AB-DTPA & Mehlich-1 \\
\hline & mg vaso-1 & $\mathrm{mg} \mathrm{kg}^{-1}$ & 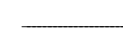 & 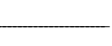 & $n^{-3}$ & - \\
\hline & & & & & & \\
\hline Resina & $-0,18^{\mathrm{NS}}$ & $0,04^{N S}$ & & & & \\
\hline DTPA & $-0,28 \mathrm{NS}$ & $-0,00^{N S}$ & $0,41^{\mathrm{NS}}$ & & & \\
\hline AB-DTPA & $0,00^{\text {NS }}$ & $0,19^{\mathrm{NS}}$ & $0,67^{*}$ & $0,67 *$ & & \\
\hline Mehlich-1 & $-0,12^{\mathrm{NS}}$ & $-0,04^{N S}$ & $0,89 * *$ & $0,89 * *$ & $0,62^{\mathrm{NS}}$ & \\
\hline Mehlich-3 & $-0,05^{N S}$ & $0,06^{N S}$ & $0,83^{*}$ & $0,83^{*}$ & $0,57 \mathrm{NS}$ & $0,89 * *$ \\
\hline \multirow[t]{2}{*}{ Resina } & $0,17^{\mathrm{NS}}$ & $0,01^{\mathrm{NS}}$ & & & & \\
\hline & \multicolumn{6}{|c|}{ Milho } \\
\hline DTPA & $0,16^{\mathrm{NS}}$ & $0,01^{\mathrm{NS}}$ & $0,80^{*}$ & & & \\
\hline AB-DTPA & $0,14^{\mathrm{NS}}$ & $-0,07^{\mathrm{NS}}$ & $0,70^{*}$ & 0,89** & & \\
\hline Mehlich-1 & $0,35^{\mathrm{NS}}$ & $-0,02^{\mathrm{NS}}$ & $0,69 *$ & 0,87** & $0,87 * *$ & \\
\hline Mehlich-3 & $0,34^{\mathrm{NS}}$ & $0,03^{N S}$ & $0,64^{*}$ & $0,85^{*}$ & $0,83 *$ & $0,94^{* *}$ \\
\hline
\end{tabular}

*; ** e NS: Significativos a 1 e 5\% de probabilidade e não significativo. 
seguinte ordem decrescente de extração foi dbservada: Mehlich-3 > Mehlich-1 =AB-DTPA > DTPA $>$ resina, para a cultura do milho, e Mehlich-3 $>A B$ DTPA > Mehlich-1 > DTPA > resina, para a soja. Esses resultados mostram, de maneira geral, que as soluções áci das empregadas foram mais eficientes em extrair o Fe do sol o do queas demais, talvez pela solubilização de parte do Fe associado às frações oxídicas do solo (Amaral Sobrinho et al., 1993). Quantoà resina, apesar deser a amplitude deextração rel ativamente el evada (Quadro 1), observou-se que a mai oria das amostras apresentou teores deFeentre 10 e $20 \mathrm{mg} \mathrm{dm}^{-3}$, mostrando a baixa presença desse elemento no sol o na forma trocável.

Embora as capacidades extrativas dos métodos tenham sido diferentes, observa-se queeles tiveram comportamentos semel hantes, fato confirmado pel os coeficientes de correlação signi ficativos obtidos entre eles, principalmente para o cultivo do milho (Quadro 3). No cultivo da soja, quando se empregou a resina como padrão, as mel hores correl ações foram obtidas com o Mehlich-1 (0,89**) e Mehlich-3 $\left(0,83^{* *}\right)$. Contudo, para o milho, todos os métodos apresentaram comportamentos parecidos com os da resina (Quadro 3).

\section{CONCLUSÕES}

1. Os métodos testados, com exceção do ABDTPA, foram eficientes em avaliar a disponibilidade de $\mathrm{Mn}$ para as plantas de soja, porém ineficientes para o milho.

2. A análise química do solo efetuada por diferentes extratores não foi eficiente para avaliar a disponibilidade deFe para as plantas demilho esoja.

3. A resina foi tão eficiente quanto o DTPA, o Mehlich-1 eo Mehlich-3 em avaliar a disponibilidade de Mn para a soja.

\section{LITE RATURA CITADA}

ABREU, C.A.; NOVAIS, R.F.; RAIJ , B. van \& RIBEIRO, A.C. Comparação de métodos químicos para avaliar a disponibilidade de manganês para a soja. R. Bras. Ci. Solo, 18:81-90, 1994a.

ABREU, C.A.; NOVAIS, R.F.; RAIJ , B. van \& RIBEIRO, A.C. Influência da reação do solo na extração de manganês por diferentes extratores químicos. R. Bras. Ci Solo, 18:91-99, 1994b.
ALMEIDA, A.M. A resina de troca iônica como extrator multiel ementar em análise de sol os para fins de fertilidade. Campinas, Instituto de Química, Universidade Estadual de Campinas, 1999. 103p. (Tese de mestrado)

AMARAL SOBRINHO, N.M.B.; COSTA, L.M.; DIAS, L.E. \& BARROS, N.F. Aplicação de resíduo siderúrgico em um Latossolo: efeitos na correção do solo e na disponibilidade de nutrientes e metais pesados. R. Bras. Ci. Solo, 17:299304, 1993.

BATAGLIA, O.C.; FURLANI, A.M.C.; TEIXEIRA, J.P.F.; FURLANI, P.R. \& GALLO, J.R. Métodos de análise de plantas. Campinas, Instituto Agronômico de Campinas, 1983. 48p. (Bol etim Técnico, 78)

DEFELIPO, B.V.; NOGUEIRA, A.V.; LOURES, E.G. \& ALVAREZV., V.H. Eficiência agronômica do lodo de esgoto proveniente de uma indústria siderúrgica. R. Bras. Ci. Solo, 15:389-393, 1991.

GOLDEBERG, S.P. \& SMITH, K.A. Soil manganese: E values, distribuition of manganese-54 among soil fractions, and effects of drying. Soil Sci. Soc. Am. J ., 48:559-564, 1984.

LINDSAY, W.L. \& NORVELL, W.A. Development of a DTPA soil test for zinc, iron, manganese, and copper. Soil Sci. Soc. Am. J ., 42:421-428, 1978.

MEHLICH, A. Determination of $\mathrm{P}, \mathrm{Ca}, \mathrm{Mg}, \mathrm{K}, \mathrm{Na}$ and $\mathrm{NH}_{4}$. Raleihg, North Carolina Soil Test Division, 1953. Não paginado

MEHLICH, A. Mehlich-3 soil test extractant. A modification of Mehlich-1 extractant. Comm. Soil Sci. Plant Anal., 15:14091416, 1984

MURAOKA, T.; NEPTUNE, A.M.L. \& NASCIMENTO FILHO, V.F. Avaliação da disponibilidade de zinco e manganês do solo para o feijoeiro. II. Manganês. R. Bras. Ci. Solo, 7:177182, 1983.

RAIJ , B. van; CANTARELLA, H.; QUAGGI O, J .A. \& FURLANI, A.M.G., eds. Recomendações de adubação e calagem para o estado de São Paulo. Campinas, Instituto Agronômico \& Fundação IAC, 1997. 285p. (Boletim 100)

RAIJ , B. van; QUAGGIO, J .A.; CANTARELLA, H.; FERREIRA, M.E.; LOPES, A.S. \& BATAGLIA, O.C. Análise química do solo para fins de fertilidade. Campinas, Fundação Cargil, 1987. 170p.

ROSOLEM, C.A.; BESSA, M.A.; AMARAL, P.G. \& PEREIRA, H.F.M. Manganês no solo, sua avaliação e toxidez de manganês em soja. Pesq. Agropec. Bras., 2792:277-285, 1992.

SOLTANPOUR, P.N.; SCHWAB, F.S. \& DEAN, L.A. A new soil test for simultaneous extraction of macro- and micronutrients in alkaline soils. Comm. Soil Sci.Plant Anal., 8:195-207, 1977. 